\title{
Boron-based donor-spiro-acceptor compounds exhibiting thermally activated delayed fluorescence (TADF)
}

\author{
Marco Stanoppi (D) and Andreas Lorbach (D) *
}

Four boron-based donor-spiro-acceptor compounds, composed of different donor moieties and borylated 2-phenylpyridines as the acceptor, were studied. Their intense photoluminescence in the solid state can be tuned by changing the donor and long emission lifetimes on the microsecond scale indicate thermally activated delayed fluorescence (TADF).

A crucial factor that determines the efficiency of organic lightemitting devices (OLEDs) is the extent to which triplet excited molecules contribute to the light emission. ${ }^{1}$ In contrast to photoexcitation, electrical excitation is based on the recombination of an electron and a hole, which predominantly generates triplet excitons (75\%). Even though purely organic phosphors are also known, ${ }^{2}$ in the absence of heavy elements with pronounced spin-orbit coupling, such triplet excitons are typically non-emissive and their energy dissipates thermally. To overcome this energy loss, heavy atoms can be installed which induce phosphorescence. ${ }^{3}$ Another strategy relies on the repopulation of an emissive singlet excited state from the longlived triplet state via reverse intersystem crossing (RISC). Both large spin-orbit coupling constants and small energy differences between these two states $\left(\Delta E_{\mathrm{ST}}\right)$ lead to an increase of the RISC rate. Therefore, small singlet-triplet gaps $\left(\Delta E_{\mathrm{ST}}<0.2\right.$ $\mathrm{eV}$ ) allow a thermal excitation from a triplet state into a singlet state even in the absence of heavy elements. ${ }^{4}$ This steady repopulation of the emissive state from a long-lived reservoir leads to thermally activated delayed fluorescence (TADF), which increases the electroluminescence quantum yield and therefore the OLED efficiency. ${ }^{5}$ Small singlet-triplet gaps can be achieved by spatially separating the frontier orbitals, ${ }^{6}$ e.g., by linking two $\pi$ conjugated systems through a spiro atom, which acts as a rigid barrier ( $c f . \mathbf{A}^{7}$ and $\mathbf{B},{ }^{8}$ Chart 1$) .{ }^{9}$ In donor-spiroacceptor compounds, the HOMO is located at the donor and

Universität Konstanz, Fachbereich Chemie, Universitätsstr. 10, 78464 Konstanz, Germany. E-mail: andreas.lorbach@uni-konstanz.de the LUMO at the acceptor. By adjusting the energy difference between these orbitals, the emission wavelength of a luminescent material can be tuned.

Borylated 2-phenylpyridines (ppy), $c f . \mathbf{B},{ }^{8} \mathbf{C},{ }^{10}$ and $\mathbf{C}^{\mathbf{F}},{ }^{11}$ are attractive acceptor moieties and transition-metal catalyzed $\left(\mathrm{Fe},{ }^{12} \mathrm{Ru},{ }^{13} \mathrm{Rh},{ }^{14} \mathrm{Ir}^{15}{ }^{15} \mathrm{Pd}^{16}\right)$ or transition-metal free ${ }^{8,17}$ borylation protocols make them readily accessible from unsubstituted ppy via $\mathrm{C}-\mathrm{H}$ bond activation. The impact of the intramolecular coordination of the nitrogen atom to the boron center is threefold: it (i) further increases the electron demand of the electron-poor ppy unit, (ii) renders the compounds stable to air and moisture, and (iii) rigidifies the structure.

Recently, Chi et al. demonstrated that the fluorinated derivative $\mathbf{C}^{\mathbf{F}}$ (Chart 1) and related compounds exhibit TADF and can be employed as high-performing OLED emitters. ${ }^{11}$ Matsuo and Yasuda attached the donor unit not to the boron center but directly to the ppy scaffold and obtained an efficient green TADF emitter. ${ }^{18}$ These are two examples of several boron-containing TADF materials that emerged in the past three years. They can be divided into compounds with tricoordinate boron, ${ }^{19}$ compounds with tetracoordinate boron, ${ }^{11,20}$ spiro compounds with tricoordinate boron in the periphery, ${ }^{21}$ and donor-spiro-acceptor compounds with a boron atom as the spiro center. ${ }^{22}$

To extend this underrepresented last class of promising TADF emitters, we decided to investigate the four compounds

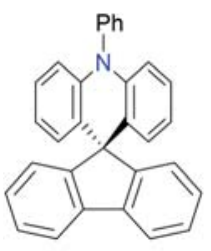

A

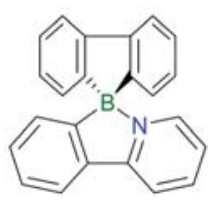

B

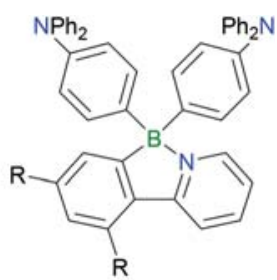

C $\mathrm{R}=\mathrm{H}$
Chart 1 Drawings of the spiro compounds $A^{7}$ and $B^{8}$ and the borylated 2-phenylpyridines $\mathrm{B}^{8} \mathrm{C}^{10}$ and $\mathrm{C}^{\mathrm{F}}$. $^{11}$ 
1-3 (Scheme 1), which allows studying the impact of (i) different donor units (1 vs. 2 vs. 3), (ii) partial fluorination (2 vs. $\left.\mathbf{2}^{\mathbf{F}}\right)$ and (iii) the presence of a heavier third-row element (1 vs. $2,2^{\mathbf{F}}, 3$ ) on the photophysical properties. A rigid spiro framework is particularly attractive in the context of borylated 2-phenylpyridines as it should efficiently suppress photoisomerization processes, which were intensively studied by Wang et al. ${ }^{23}$

For the syntheses of $\mathbf{1}, \mathbf{2}$, and $\mathbf{2}^{\mathrm{F}}$, we utilized a modified arylation protocol of Ingleson et $a l .{ }^{8}$ and obtained the three spiro compounds in yields of $72 \%, 51 \%$, and $30 \%$, respectively (Route 1, Scheme 1). Replacing the tin compound by the presumably less toxic silicon analogue led to no conversion under similar conditions and treatment of $4^{17 a}$ with the corresponding lithium, magnesium, or zinc organyls resulted in more complex product mixtures and lower yields. ${ }^{24}$ For the generation of 3 , the synthesis protocol failed since the corresponding tin precursor turned out to be unstable in the presence of $\mathrm{AlCl}_{3}$. This change in stability could be attributed to the tert-butylated arenes that are known to be readily cleaved by $\mathrm{AlCl}_{3}{ }^{25}$ Thus, 3 was generated from the organomagnesium species instead, which was obtained by lithiation of $6^{22}$ and subsequent metathesis with $\mathrm{MgBr}_{2}$ (Route 2, 33\% yield). All derivatives 1-3 are stable towards air and water and were purified by column chromatography and recrystallization, which yielded in all four cases single crystals suitable for X-ray diffractometry.

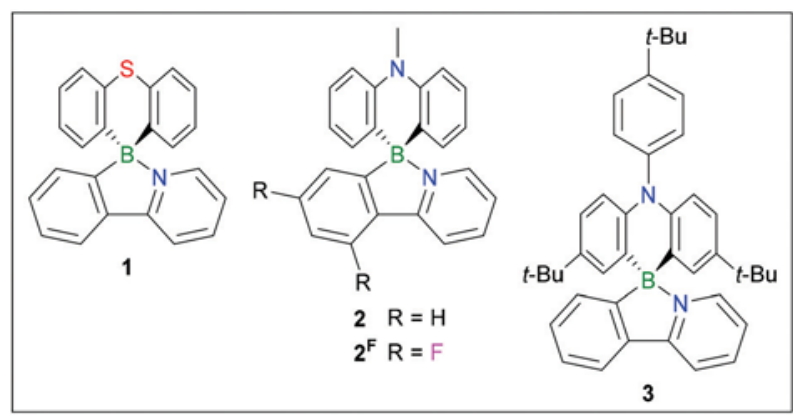

Route 1:

(for $1,2,2^{F}$ )

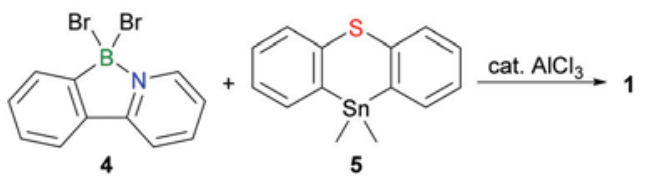

Route 2:

(for 3)

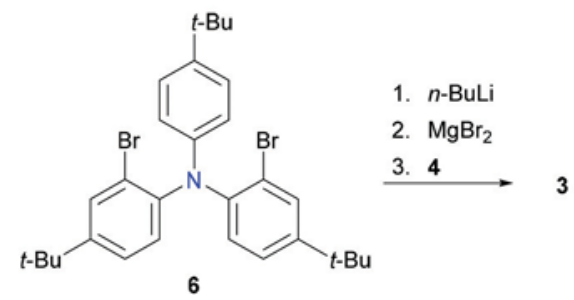

Scheme 1 Drawings of the donor-spiro-acceptor compounds 1-3 and their syntheses.
In the solid state, the borylated ppy units are planar with a maximum deviation of $0.10 \AA$ from the best planes (Fig. 1, S1, and $\mathrm{S} 2 \dagger$ ). In contrast to that, the donor units are folded along their $\mathrm{B} 1 \cdots \mathrm{S} 1$ or $\mathrm{B} 1 \cdots \mathrm{N} 2$ axis with dihedral angles between the corresponding phenylene planes of $36^{\circ}(\mathbf{1}), 31^{\circ}(\mathbf{2}), 32^{\circ}\left(2^{\mathbf{F}}\right)$, and $19^{\circ}(3)$. In contrast to that, the biphenyl unit in $\mathbf{B}$ adopts a planar conformation in the solid state. ${ }^{8}$ The folding now results in two possible orientations of the ppy with the pyridyl fragment either pointing towards the concave or the convex face of the donor moiety. In $\mathbf{1}$ the pyridyl unit is located at the concave side of the donor whereas in 2 it is located at its convex side (Fig. 1). Discrimination between the two possible isomers by X-ray crystallography solely relies on a correct assignment of the atoms $\mathrm{C} 1$ and N1, which could be problematic due to their similar atomic numbers. Swapping C1 and $\mathrm{N} 1$ in the structure refinement of $\mathbf{1}$, however, results in an increase of the $R$ value $(2.75 \%$ to $3.56 \%)$ and an unbalance of the equivalent isotropic displacement parameters $U_{\text {eq }}$ for the two atoms $\left(U_{\text {eq }}(\mathrm{C} 1) / U_{\text {eq }}(\mathrm{N} 1): 0.018 / 0.019\right.$ to $\left.0.013 / 0.025\right)$. This analysis leads to similar findings for 2 . In contrast to that, structure $2^{\mathbf{F}}$ shows positional disorder with both orientations of the fluorinated ppy present in the solid state. The additional fluoro substituents appear in the electron density map as clearly detectable maxima even for the minor component (6\%; pyridyl unit located at the concave side of the donor). 3 is disordered in the same way, even though the contribution of the minor component (35\%) is larger than in $2^{\mathbf{F}}$. Solutions of the four compounds 1-3 show only one set of signals in their ${ }^{1} \mathrm{H}$ NMR spectra at r.t. In conjunction with the results of the structure analysis, this indicates a rapid interconversion of the two isomers presumably through the inversion of the folded donor. An alternative mechanism based on a cleavage of the $\mathrm{B} 1-\mathrm{N} 1$ bond and rotation of the ppy unit along B1-C1 is unlikely since the tricoordinate boron intermediate would be prone to hydrolysis, which is not observed in wet solvents.

For $\mathbf{1}, \mathbf{2}$, and $\mathbf{2}^{\mathbf{F}}$, both isomers were identified as local minima on the potential energy surface by means of DFT calculations at the B3LYP/6-31+G* level of theory. The isomers with the pyridyl substituent located at the convex side of the donor moiety ( $c f .2$, Fig. 1) are slightly favored in all three cases with small energy differences of $0.6-1.3 \mathrm{kcal} \mathrm{mol}^{-1}$. In
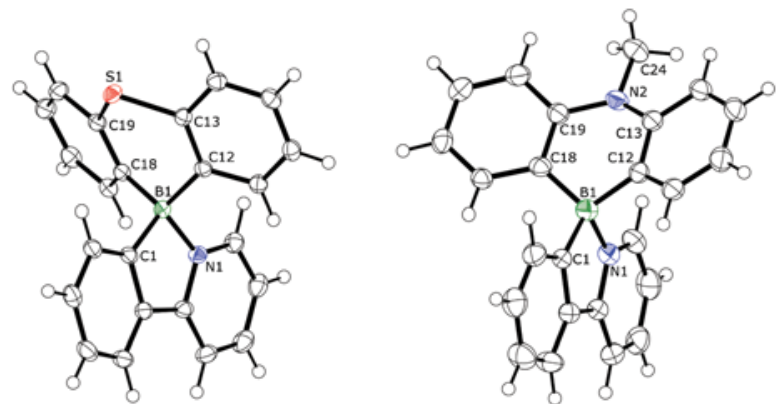

Fig. 1 Solid-state structures of 1 (left) and 2 (right). Displacement ellipsoids are drawn at the $50 \%$ probability level. 
contrast to that, optimizations of the two geometries observed in the solid-state structure of $\mathbf{3}$ only led to the same local minimum with a virtually planar donor unit.

The calculations reveal that the orientation of the ppy unit significantly alters both the HOMO and LUMO energy levels by up to $0.14 \mathrm{eV}$ (HOMOs of 1 and 2; Table S2 $\dagger$ ). This is remarkable, given that the frontier orbitals are spatially separated $\pi$ orbitals with no significant overlap (Fig. 2a). The $\sigma$ orbitals involved in the B1-N1 and B1-C1 bonds, however, display the appropriate symmetry to be part of the HOMO. The orientation of the ppy fragment now determines whether the B1-N1 or the $\mathrm{B} 1-\mathrm{C} 1 \sigma$ bond is oriented in such a way that it contributes to the HOMO most efficiently. For the B1-N1 bond, the interaction should be maximal when the torsion angle $\phi$ ( $c f$. Fig. 2b) between the B1-N1 bond and the donor phenylene unit $\mathrm{Ph}(\mathrm{C} 12)$ or $\mathrm{Ph}(\mathrm{C} 18)$ is close to $90^{\circ} .{ }^{26}$ An attachment of the pyridyl fragment at the convex face of the donor moiety leads in the calculated structures to torsion angles of $89^{\circ}(1), 87^{\circ}(2)$, and $86^{\circ}\left(2^{\mathbf{F}}\right)$, which are in line with those observed in the solid state $\left(89^{\circ}\right.$ and $90^{\circ}$ for 2 and $2^{\mathbf{F}}$, respectively). Compared to the isomers with the ppy unit oriented in the opposite direction, the calculated HOMO energies are lowered (by $0.14 \mathrm{eV}, 0.14 \mathrm{eV}$, $0.13 \mathrm{eV}$ ), the LUMO energies raised (by $0.13 \mathrm{eV}, 0.06 \mathrm{eV}, 0.06$ $\mathrm{eV}$ ), and the B1-N1 bond lengths elongated (by $0.038 \AA$, $0.026 \AA$, $0.026 \AA$ ) for 1,2 , and $2^{\mathbf{F}}$, respectively.

Upon irradiation with a UV lamp $\left(\lambda_{\text {exc }}=365 \mathrm{~nm}\right)$, powders of the four spiro compounds 1-3 exhibit intense photoluminescence ranging from green (1) to yellow ( 2 and $\left.2^{\mathbf{F}}\right)$ and orange (3) as depicted in Fig. 3. The bathochromic shift reflects an increasing strength of the donor unit $\left(\mathbf{1}<2,2^{\mathrm{F}}<3\right)$, which can be gauged by the HOMO energy level that mostly determines the HOMO-LUMO gap (Table S2 $\dagger$ ). Removal of the three tert-butyl substituents in 3 results in a lowering of the HOMO level by $0.18 \mathrm{eV}$ (Table S2†) and therefore demonstrates their strong influence on the optical properties which has been previously reported. ${ }^{22}$

In contrast to the strongly emissive solids, dilute toluene solutions of the materials show only very weak luminescence that is visibly red-shifted (Fig. 3, Table 1). A reason for the low emission intensity could be non-radiative deactivation due to the rapid interconversion of the two conformers caused by an inversion of the folded donor (see above). ${ }^{27}$ In the more polar solvents dichloromethane and acetonitrile, the emission band

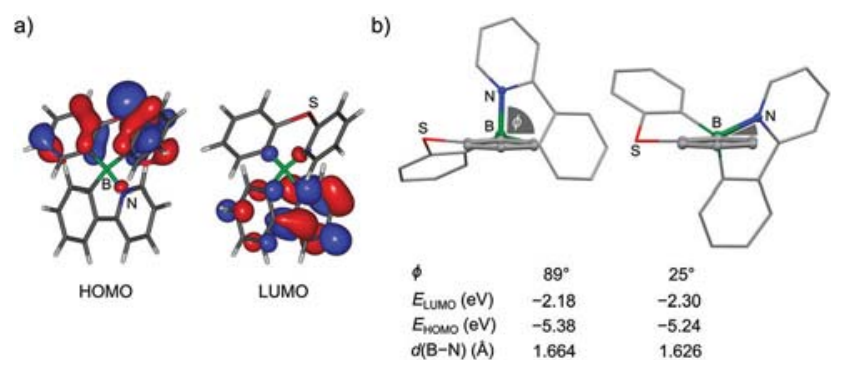

Fig. 2 Representation of the calculated frontier orbitals of 1 (a) and comparison of the two calculated isomeric structures of 1 (b).
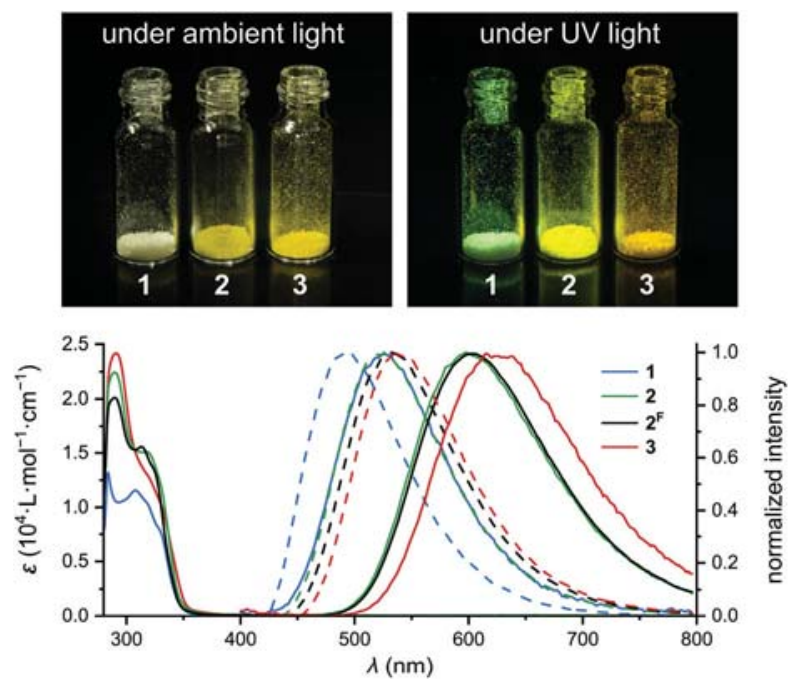

Fig. 3 Photographic pictures of powders of 1, 2, and 3 under ambient and UV light. Absorption and emission spectra of 1-3 (solid lines: toluene solutions; dashed lines: 5 wt\%-doped PMMA films).

Table 1 Photoluminescence data of 1-3

\begin{tabular}{lllll}
\hline & $\lambda_{\mathrm{em}}{ }^{a}(\mathrm{~nm})$ & $\tau^{a, b}$ & $\lambda_{\mathrm{em}}{ }^{c}(\mathrm{~nm})$ & $\Phi^{c, d}$ \\
\hline $\mathbf{1}$ & 526 & $35 \mathrm{~ns} ; 2.7 \mu \mathrm{s}$ & 492 & $11 \%$ \\
$\mathbf{2}$ & 599 & $91 \mathrm{~ns} ; 2.8 \mu \mathrm{s}$ & 524 & $33 \%$ \\
$\mathbf{2}^{\mathbf{F}}$ & 604 & $110 \mathrm{~ns} ; 1.5 \mu \mathrm{s}$ & 533 & $28 \%$ \\
$\mathbf{3}$ & 627 & $53 \mathrm{~ns}$ & 540 & $25 \%$
\end{tabular}

Conditions: ${ }^{a}$ Degassed toluene solutions. ${ }^{b}$ From TCSPC experiments with a pulsed LED $\left(\lambda_{\text {exc }}=325 \mathrm{~nm}\right) .{ }^{c}$ Doped PMMA films $(5 \mathrm{wt} \%)$, analyzed in an integrating sphere. ${ }^{d} \lambda_{\text {exc }}=340 \mathrm{~nm}$ (xenon lamp).

of 2 is further red-shifted by $1000 \mathrm{~cm}^{-1}$ and $1900 \mathrm{~cm}^{-1}$, respectively. This solvatochromism was also observed for compound $\mathbf{C}^{\mathbf{F}}$ and indicates an increase in polarity upon electronic excitation. ${ }^{11}$ Photoluminescence lifetimes of $\mathbf{1 - 3}$ in toluene solutions were obtained by means of time-correlated singlephoton counting (TCSPC) after excitation with a pulsed LED $\left(\lambda_{\text {exc }}=325 \mathrm{~nm} ; 600\right.$ ps pulse width). Analysis of the photoluminescence decay curves of 1,2 , and $2^{\mathrm{F}}$ in degassed solutions reveals the presence of two lifetime components ranging from $\tau_{1}=35 \mathrm{~ns}$ to $\tau_{1}=110 \mathrm{~ns}$ and from $\tau_{2}=1.5 \mu \mathrm{s}$ to $\tau_{2}=2.8 \mu \mathrm{s}$ (Table 1 and Fig. S5-S7†), which are similar to the emission lifetimes of the TADF material $\mathbf{C}^{\mathbf{F}}\left(\tau_{1}=75 \mathrm{~ns}\right.$ and $\left.\tau_{2}=2.7 \mu \mathrm{s}\right){ }^{11}$ Upon contact with air, the lifetimes immediately decrease considerably, e.g., 2 then shows a monoexponential decay with a lifetime of 42 ns. This quenching of triplet excited states is reversible and removal of oxygen by purging the solution with argon restores the original long-lived emission. 3 exhibits neither in aerated nor in degassed solutions delayed luminescence.

To investigate the emission behavior in the solid phase, we deposited 1-3 (5 wt\%) in a poly(methyl methacrylate) (PMMA) matrix from dichloromethane solutions inside a fluorescence 
quartz cuvette and characterized the dry samples under nitrogen within an integrating sphere. In all cases, the emission bands are blue-shifted compared to the corresponding toluene solutions (Fig. 3, Table 1), which is a result of the rigid environment in the solid phase and the less efficient stabilization of the excited state.

It is important to note that the films of $2,2^{\mathbf{F}}$, and 3 show a yellow tint in the corners of the cuvette where the material layer is thicker and the drying process slower. In line with that, an additional broad excitation band in the visible region at $400 \mathrm{~nm}\left(2\right.$ and $\left.2^{\mathbf{F}}\right)$ and $420 \mathrm{~nm}$ (3) can be detected, whereas 1 exhibits an excitation band in the near-UV at $370 \mathrm{~nm}$. At least in the case of 2 , this behavior can be attributed to an aggregation within the film based on the observation that the excitation spectrum of a powder sample superimposes well with the additional low-energy excitation band ( $c f$. Fig. $\mathrm{S} 3 \dagger)$.

Preliminary time-resolved luminescence measurements on the PMMA films indicate for 1-3 luminescence with lifetime components in the microsecond range besides one or two shorter-lived components. 1, however, stands out with an exceptionally long lifetime of $97 \mu$ s (xenon flash lamp, $\lambda_{\text {exc }}=$ $330 \mathrm{~nm} ; 400 \mathrm{~ns}$ pulse width; $c f$. Fig. $\mathrm{S} 4 \dagger$ ).

\section{Conclusions}

We fully characterized the donor-spiro-acceptor compounds 1-3 and analyzed their photoluminescence properties. In degassed solutions, $\mathbf{1}, \mathbf{2}$, and $\mathbf{2}^{\mathbf{F}}$ show TADF as indicated by a weak but long-lived emission with luminescence lifetimes on the microsecond scale. The different donor moieties directly influence the emission wavelength, which covers the range from $526 \mathrm{~nm}$ (1; weaker donor) to $627 \mathrm{~nm}$ (3; stronger donor). The fluoro substituents in $2^{\mathbf{F}}$, however, have only a minor impact on the emission properties compared to 2 . When embedded in PMMA matrices, the photoluminescence intensities of all four compounds increase considerably, reflected by quantum yields between $11 \%$ (1) and 33\% (2). All four compounds now exhibit emission lifetimes of at least several microseconds and the largest is observed for the sulfur-containing derivative $\mathbf{1}(97 \mu \mathrm{s})$. In contrast to previous TADF emitters based on borylated ppy, ${ }^{11,18} \mathbf{1}, 2$, and 3 can be synthesized from unsubstituted 2-phenylpyridine - a particularly cheap precursor. Furthermore, it is expected that the spiro architecture suppresses undesired photoisomerization processes of borylated ppy that could limit the device stability. ${ }^{10}$ We are currently designing stiffer architectures to avoid non-radiative deactivation and thus improve the photoluminescence behavior in solution.

\section{Conflicts of interest}

There are no conflicts to declare.

\section{Acknowledgements}

The authors gratefully acknowledge Dr. Michael Linseis (University of Konstanz) and Dr. Robert M. Edkins (University of Oxford) for their support in the time-resolved luminescence measurements and helpful discussions. Lisa Häfele is acknowledged for the preparation of precursors for 3. This work was supported by the state of Baden-Württemberg through bwHPC, the Deutsche Forschungsgemeinschaft DFG (project LO2167/1-1 and grant INST 40/467-1 FUGG), and the German Excellence Initiative via the Young Scholar Fund of the University of Konstanz (project 83943218).

\section{Notes and references}

1 H. Yersin, A. F. Rausch, R. Czerwieniec, T. Hofbeck and T. Fischer, Coord. Chem. Rev., 2011, 255, 2622-2652.

2 (a) S. Mukherjee and P. Thilagar, Chem. Commun., 2015, 51, 10988-11003; (b) S. Hirata, Adv. Opt. Mater., 2017, 5, 1700116.

3 (a) M. A. Baldo, D. F. O’Brien, Y. You, A. Shoustikov, S. Sibley, M. E. Thompson and S. R. Forrest, Nature, 1998, 395, 151-154; (b) P.-T. Chou and Y. Chi, Chem. - Eur. J., 2007, 13, 380-395.

4 T. J. Penfold, F. B. Dias and A. P. Monkman, Chem. Commun., 2018, 54, 3926-3935.

5 (a) H. Uoyama, K. Goushi, K. Shizu, H. Nomura and C. Adachi, Nature, 2012, 492, 234-238; (b) M. Y. Wong and E. Zysman-Colman, Adv. Mater., 2017, 29, 1605444; (c) Z. Yang, Z. Mao, Z. Xie, Y. Zhang, S. Liu, J. Zhao, J. Xu, Z. Chi and M. P. Aldred, Chem. Soc. Rev., 2017, 46, 9151016.

6 Y. Im, M. Kim, Y. J. Cho, J.-A. Seo, K. S. Yook and J. Y. Lee, Chem. Mater., 2017, 29, 1946-1963.

7 C. Poriel, J. Rault-Berthelot, S. Thiery, C. Quinton, O. Jeannin, U. Biapo, D. Tondelier and B. Geffroy, Chem. Eur. J., 2016, 22, 17930-17935.

8 D. L. Crossley, J. Cid, L. D. Curless, M. L. Turner and M. J. Ingleson, Organometallics, 2015, 34, 5767-5774.

9 (a) G. Méhes, H. Nomura, Q. Zhang, T. Nakagawa and C. Adachi, Angew. Chem., Int. Ed., 2012, 51, 11311-11315; (b) T. Nakagawa, S.-Y. Ku, K.-T. Wong and C. Adachi, Chem. Commun., 2012, 48, 9580-9582; (c) K. Nasu, T. Nakagawa, H. Nomura, C.-J. Lin, C.-H. Cheng, M.-R. Tseng, T. Yasuda and C. Adachi, Chem. Commun., 2013, 49, 10385-10387; (d) H. Ohkuma, T. Nakagawa, K. Shizu, T. Yasuda and C. Adachi, Chem. Lett., 2014, 43, 1017-1019; (e) J. Lu, Y. Zheng and J. Zhang, RSC Adv., 2015, 5, 18588-18592; (f) G. H. Lee and Y. S. Kim, Polym. Bull., 2016, 73, 24392446.

10 S. K. Mellerup, K. Yuan, C. Nguyen, Z.-H. Lu and S. Wang, Chem. - Eur. J., 2016, 22, 12464-12472.

11 Y.-J. Shiu, Y.-T. Chen, W.-K. Lee, C.-C. Wu, T.-C. Lin, S.-H. Liu, P.-T. Chou, C.-W. Lu, I-C. Cheng, Y.-J. Lien and Y. Chi, J. Mater. Chem. C, 2017, 5, 1452-1462. 
12 Y. Yoshigoe and Y. Kuninobu, Org. Lett., 2017, 19, 34503453.

13 (a) J. A. Fernández-Salas, S. Manzini, L. Piola, A. M. Z. Slawin and S. P. Nolan, Chem. Commun., 2014, 50, 6782-6784; (b) S. Okada, T. Namikoshi, S. Watanabe and M. Murata, ChemCatChem, 2015, 7, 1531-1534; (c) S. D. Sarkar, N. Y. P. Kumar and L. Ackermann, Chem. Eur. J., 2017, 23, 84-87.

14 (a) S. Kawamorita, T. Miyazaki, H. Ohmiya, T. Iwai and M. Sawamura, J. Am. Chem. Soc., 2011, 133, 19310-19313; (b) E. C. Keske, B. D. Moore, O. V. Zenkina, R. Wang, G. Schatte and C. M. Crudden, Chem. Commun., 2014, 50, 9883-9886.

15 (a) A. Ros, B. Estepa, R. López-Rodríguez, E. Álvarez, R. Fernández and J. M. Lassaletta, Angew. Chem., Int. Ed., 2011, 50, 11724-11728; (b) B. Ghaffari, S. M. Preshlock, D. L. Plattner, R. J. Staples, P. E. Maligres, S. W. Krska, R. E. Maleczka Jr. and M. R. Smith III, J. Am. Chem. Soc., 2014, 136, 14345-14348; (c) G. Wang, L. Liu, H. Wang, Y.-S. Ding, J. Zhou, S. Mao and P. Li, J. Am. Chem. Soc., 2017, 139, 91-94.

16 Y. Kuninobu, T. Iwanaga, T. Omura and K. Takai, Angew. Chem., Int. Ed., 2013, 52, 4431-4434.

17 (a) N. Ishida, T. Moriya, T. Goya and M. Murakami, J. Org. Chem., 2010, 75, 8709-8712; (b) M. Murakami, N. Ishida, T. Moriya, T. Goya, K. Morii, M. Hasegawa and Y. Arimoto, PCT Int. Pat., WO 2011/099331A1, 2011.

18 K. Matsuo and T. Yasuda, Chem. Commun., 2017, 53, 87238726.

19 (a) H. Hirai, K. Nakajima, S. Nakatsuka, K. Shiren, J. Ni, S. Nomura, T. Ikuta and T. Hatakeyama, Angew. Chem., Int. Ed., 2015, 54, 13581-13585; (b) Y. Kitamoto, T. Namikawa, D. Ikemizu, Y. Miyata, T. Suzuki, H. Kita, T. Sato and S. Oi, J. Mater. Chem. C, 2015, 3, 9122-9130; (c) K. Suzuki, S. Kubo, K. Shizu, T. Fukushima, A. Wakamiya, Y. Murata, C. Adachi and H. Kaji, Angew. Chem., Int. Ed., 2015, 54, 15231-15235; (d) T. Hatakeyama, K. Shiren, K. Nakajima, S. Nomura, S. Nakatsuka, K. Kinoshita, J. Ni, Y. Ono and T. Ikuta, Adv. Mater., 2016, 28, 2777-2781; (e) Y. Liu, G. Xie, K. Wu, Z. Luo, T. Zhou, X. Zeng, J. Yu, S. Gong and C. Yang, J. Mater. Chem. C, 2016, 4, 4402-4407; (f) Y. Kitamoto, T. Namikawa, T. Suzuki, Y. Miyata, H. Kita, T. Sato and S. Oi, Tetrahedron Lett., 2016, 57, 4914-4917; $(g)$ Y. Kitamoto, T. Namikawa, T. Suzuki, Y. Miyata, H. Kita, T. Sato and S. Oi, Org. Electron., 2016, 34, 208-217; (h) Y. H. Lee, S. Park, J. Oh, J. W. Shin, J. Jung, S. Yoo and M. H. Lee, ACS Appl. Mater. Interfaces, 2017, 9, 2403524042; (i) T.-L. Wu, M.-J. Huang, C.-C. Lin, P.-Y. Huang, T.-Y. Chou, R.-W. Chen-Cheng, H.-W. Lin, R.-S. Liu and C.-H. Cheng, Nat. Photonics, 2018, 12, 235-240; (j) D.-G. Chen, T.-C. Lin, C.-L. Chen, Y.-T. Chen, Y.-A. Chen, G.-H. Lee, P.-T. Chou, C.-W. Liao, P.-C. Chiu, C.-H. Chang, Y.-J. Lien and Y. Chi, ACS Appl. Mater. Interfaces, 2018, 10, 12886-12896.

20 (a) C. A. DeRosa, J. Samonina-Kosicka, Z. Fan, H. C. Hendargo, D. H. Weitzel, G. M. Palmer and C. L. Fraser, Macromolecules, 2015, 48, 2967-2977; (b) Y.-J. Shiu, Y.-C. Cheng, W.-L. Tsai, C.-C. Wu, C.-T. Chao, C.-W. Lu, Y. Chi, Y.-T. Chen, S.-H. Liu and P.-T. Chou, Angew. Chem., Int. Ed., 2016, 55, 3017-3021.

21 (a) M. Numata, T. Yasuda and C. Adachi, Chem. Commun., 2015, 51, 9443-9446; (b) Y.-J. Lien, T.-C. Lin, C.-C. Yang, Y.-C. Chiang, C.-H. Chang, S.-H. Liu, Y.-T. Chen, G.-H. Lee, P.-T. Chou, C.-W. Lu and Y. Chi, ACS Appl. Mater. Interfaces, 2017, 9, 27090-27101.

22 B. M. Bell, T. P. Clark, T. S. De Vries, Y. Lai, D. S. Laitar, T. J. Gallagher, J.-H. Jeon, K. L. Kearns, T. McIntire, S. Mukhopadhyay, H.-Y. Na, T. D. Paine and A. A. Rachford, Dyes Pigm., 2017, 141, 83-92.

23 (a) Y.-L. Rao, H. Amarne, S.-B. Zhao, T. M. McCormick, S. Martić, Y. Sun, R.-Y. Wang and S. Wang, J. Am. Chem. Soc., 2008, 130, 12898-12900; (b) Y.-L. Rao, H. Amarne and S. Wang, Coord. Chem. Rev., 2012, 256, 759-770; (c) S. K. Mellerup, L. Häfele, A. Lorbach, X. Wang and S. Wang, Org. Lett., 2017, 19, 3851-3854; (d) S. K. Mellerup, C. Li, T. Peng and S. Wang, Angew. Chem., Int. Ed., 2017, 56, 6093-6097; (e) J. Wang, B. Jin, N. Wang, T. Peng, X. Li, Y. Luo and S. Wang, Macromolecules, 2017, 50, 4629-4638.

24 Optimizations of the reaction conditions were carried out for the synthesis of 2 .

25 M. Tashiro, Synthesis, 1979, 921-936.

26 We define the angle $\phi$ as the dihedral angle between a phenylene unit of the donor (e.g., $\mathrm{Ph}(\mathrm{C} 12)$, Fig. 1) and the plane spanned by its ipso-carbon atom and the $\mathrm{BN}$ fragment attached to it (e.g., C12, B1, and N1).

27 H. Güsten and R. Meisner, J. Photochem., 1983, 21, 53-60. 